\section{Oral and intranasal sedation}

Oral sedation is useful for patients who are unable to accept cannulations due to needle phobia or a learning disability. The sedation produced may be adequate for the dental procedure to be carried out or it may achieve sufficient relaxation to allow cannulation for intravenous sedation. The most commonly used drug is midazolam which can also be administered intranasally. These routes produce less predictable sedation, as the drug is not titrated to individual requirements. Oral and intranasal sedation must only be administered by practitioners who are also trained and experienced in the use of intravenous sedation.

\section{Summary}

Conscious sedation is fundamental to providing dental care and all anxious patients should have access to dentistry in this way. Sedation also

\title{
Fear-free dentistry
}

\section{by Dr Jennifer Pinder*}

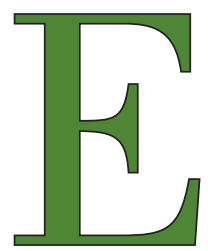

stimates vary but about $11 \%$ of the population avoid going to the dentist even when in extreme pain. Providing dentistry that helps people to get rid of their fears and which doesn't create new anxieties is a challenge for dental care professionals (DCPs). Skilled communication, pain control and anxiety management are the key skills needed for treating all patients, but especially for dental phobics.

The following is a message that I received by email that illustrates a severe dental phobia:

'My wife is currently taking antibiotics for a dental infection and is in pain but there is no way she will go to any dentist. The last time she had such an infection it took 18 months of pain and all the antibiotics she could twist our GP's arm for before she would attend a dentist for an extraction which was very traumatic (she never went back for a follow up check).

She now has one in another tooth and just the mere mention of going to a dentist reduces my wife to tears. I am at a loss to know what our next move should be and am interested to find out if you feel there is anything you can do to help.

Her history briefly is, she would regularly go to the dentist for treatment under a general anaesthetic but once that became unavailable and following a bad experience with deep sedation, she now insists if there is no general anaesthetic available she will put up with the pain. Our GP referred her to Guy's School of Dentistry but she left in tears and never went back after being flatly denied a general anaesthetic. She has had other

\footnotetext{
* Jennifer is a general dental practitioner with a special interest in helping phobic patients. She sat on the General Dental Council for 17 years until 2001 and was Vice-Dean of the Faculty of General Dental Practitioners of the Royal College of Surgeons in 1996.
}

surgery and frankly was unperturbed by it; our children were delivered by caesarean during which she was conscious and she was totally comfortable with that, however dentistry seems to hold a special fear for her. Can you suggest how we can best proceed to get her treated?'

There are many reasons that dental phobias develop and an in depth assessment with the patient is invaluable for developing a successful treatment plan.

Some people can't identify exactly why the problem began, but many others remember traumatic and difficult experiences vividly. The emotions associated with these events are easily re-activated and have been described as Post Traumatic Dental Stress Disorder (PTSD).

Examples of common causes are:

Injection phobia - This may be a general fear of injections anywhere in the body, only of dental injections, or fear of fainting.

Fear of pain - Painful treatment is avoidable and anxious patients commonly report that local anesthetics have not worked properly but they have not been taken seriously.

Gagging problems - Patients avoid treatment due to having an exaggerated gag reflex. This causes difficulties for dental professionals and embarrassment for the person being treated.

Complex problems - There may just be a simple dental phobia but this may be combined with other psychological problems such a generalised anxiety disorder (GAD), depression, or other mental disorders.

Physical and/or sexual abuse - This can be an undisclosed problem which can lead to dental avoidance, but presents particular challenges for the dental team.

There are many strategies and techniques to help people have the dental care that they need. Some are based on sound psychological principles such as systematic desensitisation. There are pharmacological aids ranging helps non-anxious people cope with unpleasant treatment such as oral surgery.

\section{Further reading}

Craig D, Skelly D. Practical conscious sedation. London: Quintessence Publishing Co Ltd, 2004.

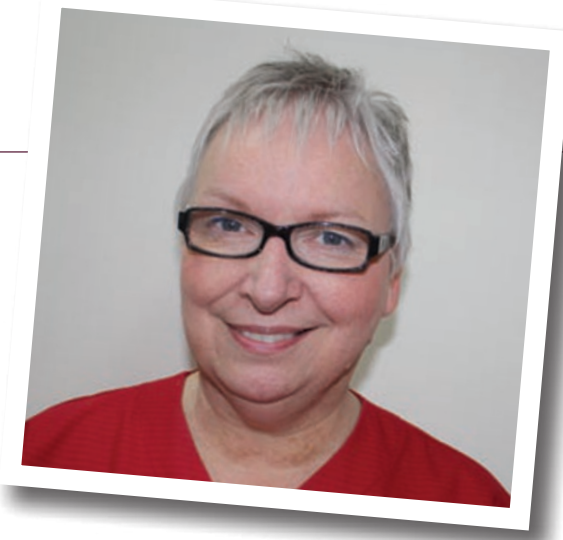

from sedative tablets or relative analgesia to anaesthetist-delivered sedation. Some patients will only accept treatment under a general anaesthetic (GA). There are also some new gadgets around such as the dental button, the Nucalm system and the Wand digital injection system.

\section{The role of dental care professionals}

Dental hygienists and therapists regularly have anxious patients to treat and dental hygiene procedures are more feared and avoided than is acknowledged. The regular discussion and acknowledgement of good pain control should be routine. Relative analgesia is going to be a part of the range of tools available after training for these DCPs.

Dental nurses also have a role to play in supporting the patient, being empathetic, and communicating well with them. Often a person will tell the nurse something that they don't tell the dentist.

DCPs could play a bigger role in the initial assessment of the patient before the clinical examination.

Successful treatment of this group of patients brings a great deal of professional satisfaction, and the knowledge that you have made a difference to the quality of a person's life.

Jennifer and Carole, along with vocal psychologist Fergus McClelland, will be presenting a new BDA Training essentials course, 'Fear-free dentistry: managing phobic patients' which is due to take place on Thursday 2 December, and repeated again on Friday 8 April 2011 in London. Visit Www.bda.org/training or call the BDA events team on 02075634590 for further details. 